\title{
Velocity profile and depth-averaged to surface velocity in natural streams: A review over a large sample of rivers
}

\author{
Alexandre Hauet ${ }^{1, *}$, Thomas Morlot ${ }^{2}$ and Léa Daubagnan ${ }^{2}$ \\ ${ }^{1}$ EDF - DTG, 21 avenue de l'Europe, 38000 Grenoble, France \\ ${ }^{2}$ EDF - DTG, 14 rue Paul Mesplé, 31100 Toulouse, France
}

\begin{abstract}
For measuring flood discharge with non-intrusive methods, one should know the depth-averaged to surface velocity ratio $\alpha$. In this paper, a large sample of gauging (3611 gaugings over 176 sites of EDF-DTG) realized with a current meter are analyzed to compute $\alpha$ with three methods: fitting of a log- and a power-law and using the measured surface velocity. Results show that the three methods give very similar results, with a mean value of $\alpha$ of about 0.8 , with few dispersion $\left(5^{\text {th }}\right.$ and $95^{\text {th }}$ percentile values being about 0.7 and 0.9 ). A link between $\alpha$ and the water depth is established, as $\alpha$ increases with the hydraulic radius. The link between $\alpha$ and the bed roughness, or between $\alpha$ and the relative roughness $(\mathrm{Rh} / \mathrm{d} 50)$ is not clear. Results only shows that the mean value of $\alpha$ is 0.8 for natural rivers (sandy, pebbly or boulders rivers), and 0.9 for artificial concrete channels.
\end{abstract}

\section{Introduction}

\subsection{EDF-DTG hydrometric network}

EDF (Electricité De France) is the world's largest electricity generator, with an installed capacity of about $140 \mathrm{GWhe}$. In order to safely operate the plants, to optimize the natural resources and to fulfill ecological requirement, EDF-DTG has installed, since 1946, a sensor network dedicated to the monitoring of hydro-climatologic parameters. This network includes about 300 rain gauges stations (mostly tipping bucket gauges); about 40 snow gauges (snow water equivalent measurement using cosmic ray gauge) and about 300 hydrometric stations. As illustrated on Figure 1, this sensor network is mainly located on the mountainous regions of France (Alps, Pyrenees and Massif Central), at high altitude, on small rivers with important slopes.

For a vast majority of EDF's hydrometric stations, the discharge is not directly measured: in situ sensors measure water stage continuously, which is converted into discharge using a stage-discharge relationship, so called rating curve. The rating curve is a hydraulic model that must be parameterized using direct discharge measurements, so called gaugings.

\footnotetext{
*Corresponding author: alexandre.hauet@edf.fr
} 


\subsection{Non-intrusive methods for flood discharge measurement}

Measuring flood discharge using conventional hydrometric methods is impossible, especially for mountainous rivers [1]. High velocities and floating objects makes the river too dangerous to be directly gauged by human operators. Using current meters or Acoustic Doppler Current Profilers (ADCP) in such extreme conditions is too risky and the high suspended sediment load avoids using tracer dilution methods. Riverbed instability due to rapid scouring and deposition during flooding makes measurement of cross-section bathymetry very difficult. As a consequence, the rating curves must be extrapolated for high flows. Even if this extrapolation is based on hydraulic assumptions, it brings a lot of uncertainty and the flood discharge series can be unreliable, impacting drastically flood forecasting, the sizing of structures like dam spillways, and so people's safety [2].

Non-intrusive methods are a promising alternative allowing measurement of flood flow. Surface velocities can be measured using Radar Doppler [3] or image-based methods like Large-Scale Particle Image Velocimetry [4, 5], Particle Tracking Velocimetry [6] or SpaceTime Image Velocimetry [7]. As remote measurements only measure surface velocity, assumptions regarding the link between surface and depth-averaged velocity have to be made in order to compute discharge for a cross-section of known bathymetry. A simple and convenient method is the use of a velocity coefficient $\alpha$ defined as the depth-average velocity to surface velocity ratio. A default value of $\alpha=0.85$ is commonly chosen for river flow [1, 4], assuming a logarithmic vertical velocity distribution and typical bed roughness. Abundant literature exists on the theory of velocity profile in rivers, but a review of in situ measurements over a large sample of rivers was missing: in this study, a large sample of gaugings is used to experimentally compute $\alpha$ using three different methods.

\section{Data set of discharge measurements}

EDF-DTG realizes about 900 discharge measurements (gauging) per year. 30\% of those measurements are realized using current meters (mechanical, acoustic Doppler or electromagnetic instruments) with the mid-section method [1], following the prescriptions of international standards like Iso 748 [8]. The specific protocol used at EDF-DTG imposes to measure at least 5 velocities per vertical, including a measurement as close to the free surface as possible (typically $3 \mathrm{~cm}$ below the free-surface). Each velocity measurement usually lasts 30 seconds. This protocol allows a good representation of the vertical distribution of velocity, and a good knowledge of the surface velocity. ADCP measurements are not used in that study because velocities close to the free surface cannot be measured due to the blanking distance of about $15 \mathrm{~cm}$.

A dataset of 3611 EDF-DTG's discharge measurement using current meters is used in that study. They have been realized over 176 hydrometric stations in France (see Figure 1) covering a large sample of rivers, from small mountain streams to large plain rivers, as illustrated in Table 1.

Table 1. Characteristics of the studied sites. Q10 and Q90 are the $10^{\text {th }}$ and $90^{\text {th }}$ percentile.

\begin{tabular}{|c|c|c|c|c|c|}
\hline & Min & Max & Mean & Q10 & Q90 \\
\hline Discharge (m3/s) & 0.01 & 863 & 8.45 & 0.25 & 10.2 \\
\hline Width B (m) & 0.49 & 90.3 & 13.1 & 3.80 & 24.8 \\
\hline Hydraulic radius Rh (m) & 0.02 & 5.36 & 0.40 & 0.18 & 0.63 \\
\hline Aspect ratio B/Rh & 2.43 & 165 & 35.2 & 14.0 & 64.0 \\
\hline
\end{tabular}




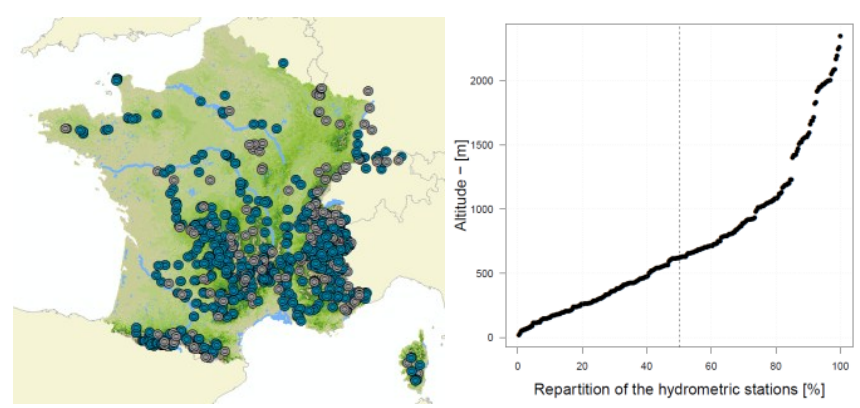

Fig. 1. Location of the 176 hydrometric stations studied (left); distribution of the altitude of the studied stations (right).

Discharges ranges from 0.01 to $863 \mathrm{~m}^{3} / \mathrm{s}$, with an averaged value of $8.45 \mathrm{~m}^{3} / \mathrm{s}$. Flood discharges were measured using cableways or hydrometric trucks. The studied rivers are mainly small to medium rivers as $90 \%$ of discharge, width and hydraulic radius are smaller than $10.2 \mathrm{~m}^{3} / \mathrm{s}, 24.8 \mathrm{~m}$ and $0.63 \mathrm{~m}$ respectively.

\section{Computation of the depth-average to surface velocity ratio}

\subsection{Theoretical background}

\subsubsection{Log-law and power-law velocity profile}

Following the law of the wall, the velocity profile in the inner region follows a log-law like:

$$
\frac{u}{U^{*}}=\frac{1}{k} \ln \left(\frac{z}{z_{0}}\right)
$$

where $u$ is the local, averaged, streamwise velocity, $U^{*}$ is the shear velocity, $k$ is the von Karman constant $(k \approx 0.4), z$ is the distance from origin of vertical velocity profile and $z_{0}$ is a hydraulic roughness parameter describing the characteristic hydraulic friction length. [9] shows that this equation can also be applied in the outer region, over the whole flow depth, with $z / z_{0}$ ranging from 3 to $10^{3}$. As shown by [10], the depth-averaged to surface velocity ratio $\alpha$ computed with typical values of $h / z_{0}$ in natural streams $\left(10^{2}-10^{4}\right)$ ranges $0.79-0.89$, with a central value close to 0.85 for $h / z_{0}=10^{3}$.

For computation convenience, the velocity profile can also be expressed as a power law like:

$$
u(z)=a \cdot\left(z-z_{0}\right)^{1 / m}
$$

where $a$ is a coefficient and $1 / m$ is referred to as the power-law index.

\subsubsection{Profiles not following a theoretical law}

The natural streams velocity profiles do not always fit a log- or power-law over the whole flow depth. As noted by [10] or [11] for large rivers with uniform flow distribution, the $\log$ profile is well adapted for $z / h<0.7$ (with $h$ the water depth), and velocities are roughly constant for $z / h>0.7$. More, singularities in the channel morphology, wind effect or the presence of aquatic vegetation can affect the velocity profile so that a log- or power-law is not applicable. 


\subsection{Velocity profile analysis method}

For the 3611 gaugings of the database, verticals of measurement realized close to the banks were removed to avoid side effects producing velocity profiles which are nor representative of the main flow. For each gauging, the depths and velocities of every verticals are normalized with the verticals' total depth and averaged velocity respectively, so as one velocity profile is produced for one gauging using all the verticals of the gauging. Then, three methods (log-law, power-law and surface velocity) are applied to compute $\alpha$ for each gauging.

\subsubsection{Log-law}

The statistical adjustment method of [9] is applied to fit a log-law (eq. 1) on the normalized measurements. The normalized profiles are fitted using $k \cdot u_{\text {normalized }}=f\left(\ln \left(z_{\text {normalized }}\right)\right.$, thus allowing calculation of $U^{*}$ (the slope of the regression line) and $z_{0}$ (the intercept of the regression line being $\left.-U^{*} \cdot \ln \left(z_{0}\right)\right)$. The value of the normalized velocity for $z_{\text {normalized }}=1$ is equal to $1 / \alpha_{\log }$.

\subsubsection{Power-law}

The normalized velocity profiles are fitted as $\ln \left(u_{\text {normalized }}\right)=\ln (a)+1 / m \cdot \ln \left(z_{\text {normalized }}{ }^{-}\right.$ $\left.z_{\text {onormazlized }}\right) . z_{0}$ is adjusted in order to maximize the correlation coefficient of the fit. The value of the normalized velocity for $z_{\text {normalized }}=1$ is equal to $1 / \alpha_{\text {power }}$.

\subsubsection{Measured surface velocity}

As the specific gauging protocol of EDF-DTG imposes a measurement as close to the freesurface as possible $u_{\text {surf }}$ (generally $3 \mathrm{~cm}$ below the free-surface), the averaged value of normalized $u_{\text {surf }}$ for a gauging is equal to $1 / \alpha_{\text {surf }}$. Figure 2 illustrates the computation of $\alpha$ with the three methods for two different gaugings. Each graph represent the normalized velocity and depth of all the measurement of a gauging.

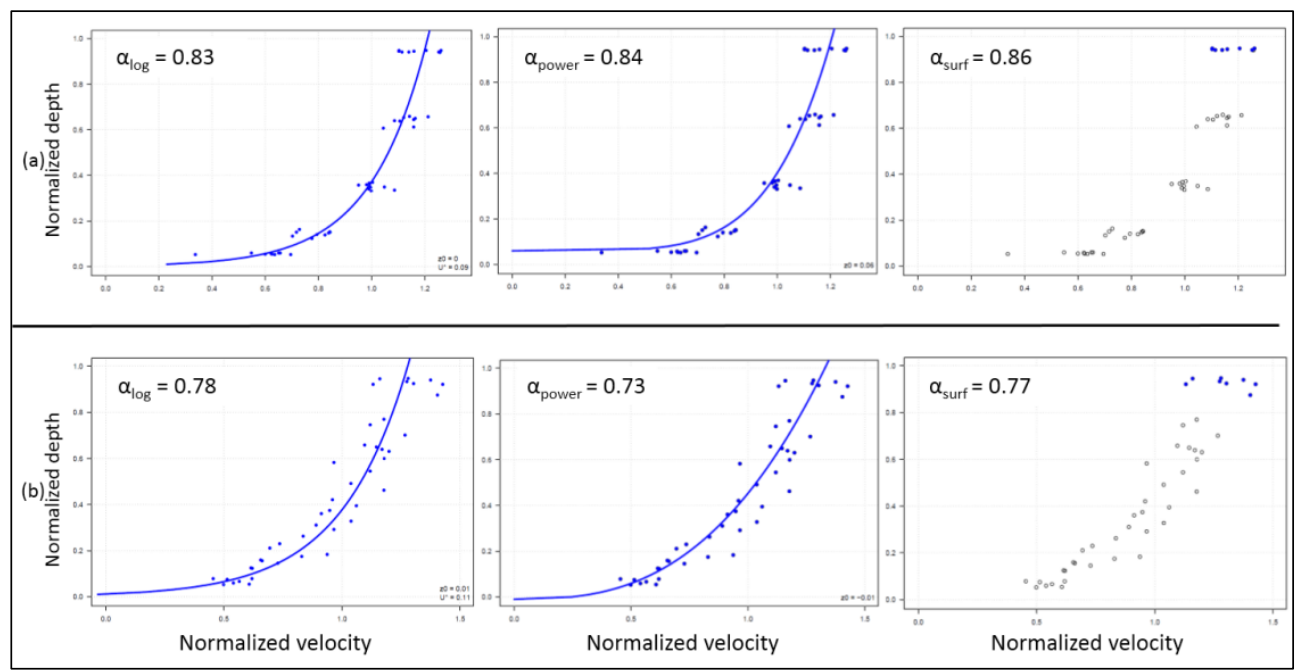

Fig. 2. Example of computation of $\alpha$ with the three methods (left: $\log$, middle: power, right: surface) and for two different gauging. 


\section{Results}

\subsection{Overall results}

As illustrated in Table 2 over the 3611 gaugings, the computed values of $\alpha$ with the three methods are quite similar with mean values of $0.81,0.79$ and 0.83 for $\alpha_{\log }, \alpha_{\text {power }}$ and $\alpha_{\text {surface }}$ respectively. Those values are consistent with the usually accepted value of 0.85 and are in very good agreements with the experimental results of [3]. Table 2 shows that the dispersion of the results is limited, as $90 \%$ of the values range from 0.73 to 0.9 for $\alpha_{\log }$, from 0.67 to 0.91 for $\alpha_{\text {power }}$ and from 0.71 to 0.95 for $\alpha_{\text {surface }}$.

Table 2. Statistics on the values of $\alpha$ computed with the three methods (log, power and surface).

\begin{tabular}{|l|l|l|l|l|l|}
\hline & \multicolumn{1}{|c|}{ Min } & \multicolumn{1}{|c|}{ Max } & Mean & Q5 & Q95 \\
\hline$\alpha_{\log }$ & 0.48 & 1 & 0.81 & 0.73 & 0.9 \\
\hline$\alpha_{\text {power }}$ & 0.36 & 0.99 & 0.79 & 0.67 & 0.91 \\
\hline$\alpha_{\text {surface }}$ & 0.32 & 2.3 & 0.83 & 0.71 & 0.95 \\
\hline
\end{tabular}

Figure 3 shows the boxplots for the three methods, including the extreme values. $\alpha_{\text {surface }}$ shows more dispersion and some extreme values ranging from 0.32 to 2.3 .

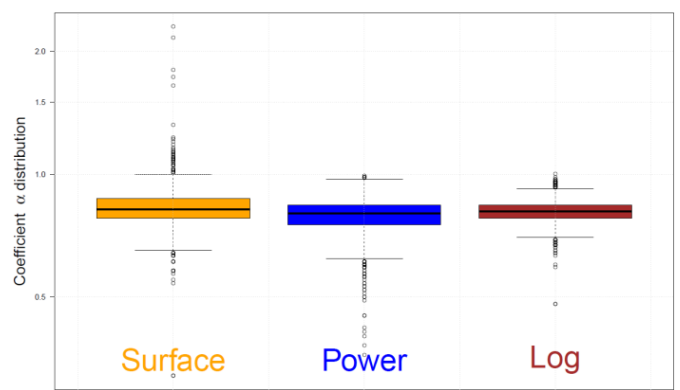

Fig. 3. Boxplots of the values of $\alpha$ computed with the three methods (surface, power and log).

The values of $\alpha_{\text {surface }}$ greater than 1 are related to wind affected flows, where the velocity close to the free surface is slowed down, as illustrated in Figure 4.

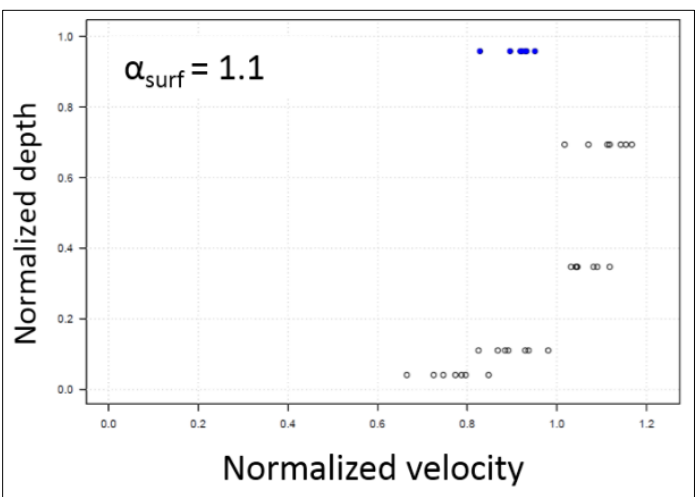

Fig. 4. Velocity profile slowed down by the wind. 


\subsection{Link between $\alpha$ and the morphology of the rivers}

When measuring a flood event using a non-intrusive method, it is impossible to have a direct measurement of the vertical distribution of the velocity. One should deduce the value of $\alpha$ from characteristics of the river.

\subsubsection{Evolution of $\alpha$ with the water depth}

Considering the literature, $\alpha$ is supposed to get close to 1 when the water depth increases and when the roughness decreases. Figure 5 shows the evolution of $\alpha_{\log }$ and $\alpha_{\text {surf }}$ with the hydraulic radius $R h$. The results for $\alpha_{\text {power }}$ are very similar to $\alpha_{\log }$, and are not presented in Figure 5. $\alpha_{\log }$ and $\alpha_{\text {surf }}$ show the same behavior: they increase with $R h$. For very shallow water $(0 \mathrm{~m}<R h<1 \mathrm{~m}) \alpha$ is close to 0.8 and it increases up to 0.9 for a $R h$ of $5 \mathrm{~m}$. The trend is linear, as illustrated on Figure 5.

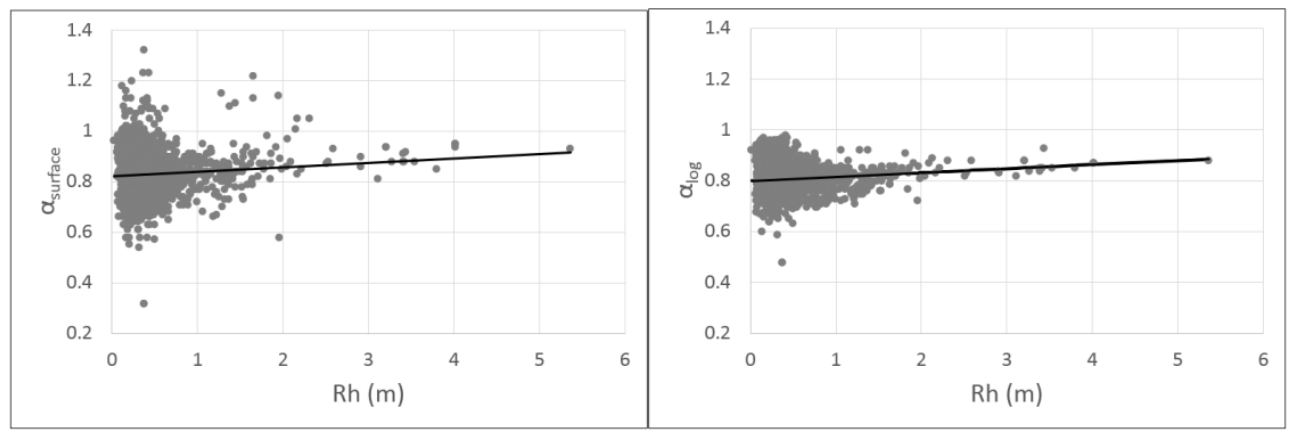

Fig. 5. Evolution of $\alpha_{\log }$ (left) and $\alpha_{\text {surf }}$ (right) with the hydraulic radius $\mathrm{Rh}$.

As the studied rivers have relative small sizes (mean $R h=0.4 \mathrm{~m}$, mean width $=13 \mathrm{~m}$ ), this can explain why the values computed for $\alpha$ over the whole sample (see Table 1) are slightly lower than the usual 0.85 used in a lot of studies.

\subsubsection{Evolution of $\alpha$ with the aspect ratio}

Figure 6 illustrates the link between $\alpha$ and the aspect ratio $B / R h$. The results for $\alpha_{\text {power }}$ are very similar to $\alpha_{\log }$, and are not presented. $\alpha_{\log }$ and $\alpha_{\text {surf }}$ seem to slightly decrease when $B / R h$ increases, but the trend is not very pronounced.

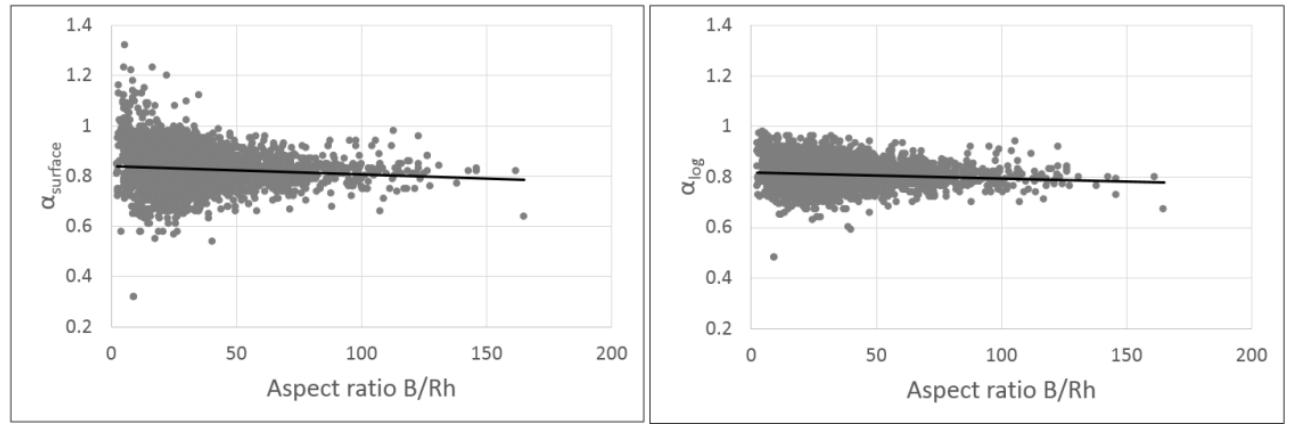

Fig. 6. Evolution of $\alpha_{\log }\left(\right.$ left) and $\alpha_{\text {surf }}$ (right) with the aspect ratio $B / R h$. 


\subsubsection{Evolution of $\alpha$ with the bed roughness}

For each of the 3611 gaugings studied, the bed roughness was estimated according to four categories: concrete channel, sandy river, pebbly river and boulders river, with a median particle size $d_{50}$ of $0.001,0.01,0.1$ and 1 meter respectively. Figure 7 shows a boxplot of $\alpha_{\text {surface }}$ computed for each station (the results for $\alpha_{\text {power }}$ and $\alpha_{\log }$ are similar and not presented in the figure). Values of $\alpha_{\text {surface }}$ greater than 2 are not shown in Figure 7 for clarity reason. The stations are sorted according to the bed roughness.

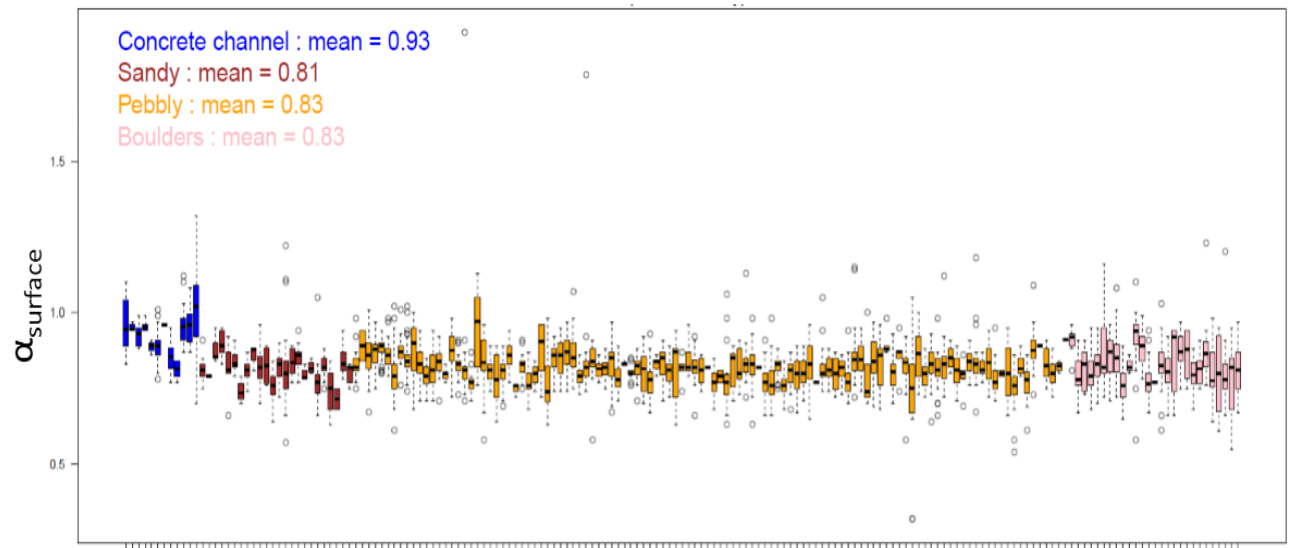

Fig. 7. Boxplots of $\alpha_{\text {surface }}$ computed for each station, sorted according to the bed roughness. Values of asurface greater than 2 are not shown for clarity reason.

For sandy, pebbly and boulders rivers, the averaged value of $\alpha$ equals about $0.8\left(5^{\text {th }}\right.$ and $95^{\text {th }}$ percentiles values being 0.71 and 0.88 respectively), with a lit bit more dispersion for the boulders rivers. The measurements realized for concrete channels have an averaged value of $\alpha$ of about $0.9\left(5^{\text {th }}\right.$ and $95^{\text {th }}$ percentiles values being 0.82 and 0.99 respectively).

Figure 8 shows the variation of $\alpha_{\text {surface }}$ with the relative roughness defined as the ratio between the hydraulic radius and the median particle size, $R h / d_{50}$. Values of $\alpha_{\text {surface }}$ greater than 2 are not shown in Figure 8 for clarity reason. $\alpha$ has a mean value of $0.8\left(5^{\text {th }}\right.$ and $95^{\text {th }}$ percentiles values being 0.71 and 0.88 , respectively) for relative roughness less than 1000 . For greater relative roughness $\left(1000<R h / d_{50}<10000\right)$, the mean value of $\alpha$ is $0.9\left(5^{\text {th }}\right.$ and $95^{\text {th }}$ percentiles values being 0.8 and 0.99 , respectively).

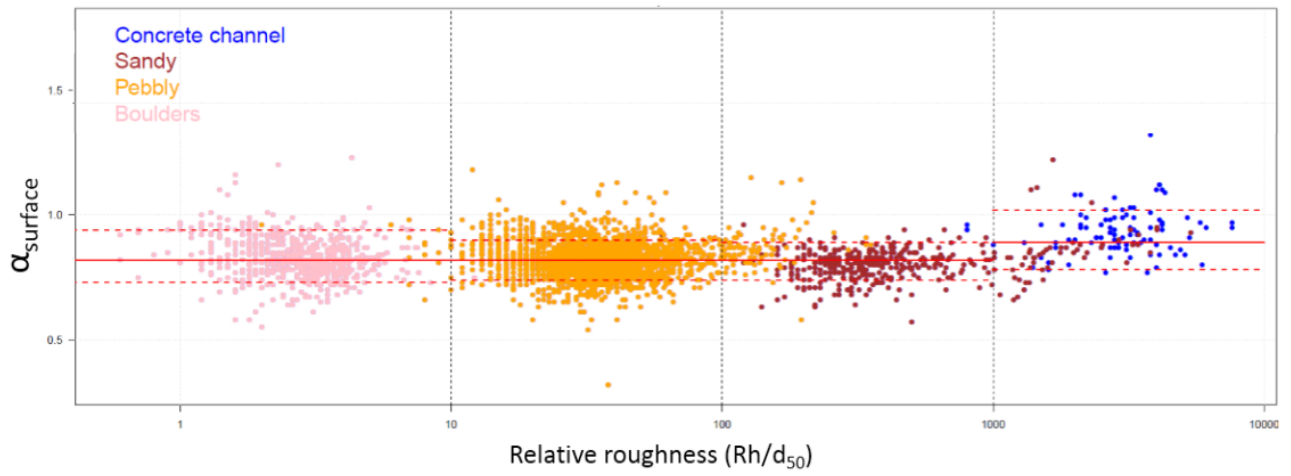

Fig. 8. Boxplots of $\alpha_{\text {surface }}$ computed for each station, sorted according to the bed relative roughness $\left(\mathrm{Rh} / \mathrm{d}_{50}\right)$. Values of $\alpha_{\text {surface }}$ greater than 2 are not shown for clarity reason. 


\section{Conclusions}

In that paper, a large sample of 3611 gaugings realized on 176 different hydrometric stations is used to compute the depth-averaged velocity to surface velocity ratio $\alpha$. For each of those gauging, three methods are used for computing $\alpha$ : fitting a log-law, a power-law, and using the measured velocities close to the free-surface.

Results show that the three computation methods give very similar results, with a mean value of $\alpha$ of about 0.8 . This value is slightly lower than the usual 0.85 used in a lot of studies, this can be explained by the relative small size of the studied rivers (mean $R h=0.4 \mathrm{~m}$, mean width $=13 \mathrm{~m}$ ). The dispersion of the computations is quite small, $5^{\text {th }}$ and $95^{\text {th }}$ percentile values being about 0.7 and 0.9 .

A link between $\alpha$ and the water depth is established, as $\alpha$ increases with the hydraulic radius $R h$. For very shallow water $(0 \mathrm{~m}<R h<1 \mathrm{~m}) \alpha$ is close to 0.8 and it increases up to 0.9 for a $R h$ of $5 \mathrm{~m}$, following a linear trend.

The link between $\alpha$ and the bed roughness, or between $\alpha$ and the relative roughness $(R h / d 50)$ is not clear. Results only shows that the mean value of $\alpha$ is 0.8 for natural rivers (sandy, pebbly or boulders rivers), and 0.9 for artificial concrete channels.

This study brings rules of thumbs for applying a depth-averaged velocity to surface velocity ratio to a non-intrusive measurement:

- For natural rivers :

$\circ$ For water depth less than 2 meters: consider using $\alpha=0.8$ with an uncertainty of about $+/-15 \%$ at $90 \%$ confidence level.

○ For greater water depth, consider using $\alpha=0.9$ with an uncertainty of about $+/-15 \%$ at $90 \%$ confidence level.

- For artificial concrete channel, consider using $\alpha=0.9$ with an uncertainty of about $+/-15 \%$ at $90 \%$ confidence level.

\section{References}

1. S.E. Rantz 1982, Measurement and computation of streamflow, (USGS Water Supply Paper, 1982)

2. M. Lang, K. Pobanz, B. Renard, E. Renouf, E. Sauquet, Hydrological Sciences Journal, 55 (6), 883-898 (2010). doi: 10.1080/02626667.2010.504186. 9

3. M. Welber, J. Le Coz, J. Laronne, G. Zolezzi, D. Zamler, G. Dramais, A. Hauet, M. Salvaro, Water Resources Research, 52(2), 1108-1126, (2016). doi: 10.1002/2015WR017906

4. M. Muste, I. Fujita, A. Hauet, Water Resour. Res., 44, (2008). doi:10.1029/2008WR006950

5. A. Hauet, A. Kruger, W.F. Krajewski, A. Bradley, M. Muste, J.-D. Creutin, M. Wilson, Journal of Hydrologic Engineering, 13(2), 105-110, (2008)

6. A. Patalano, C. Marcelo García, A. Rodríguez, Computers \& Geosciences, 109, 323 330, (2017). doi: 10.1016/j.cageo.2017.07.009

7. I. Fujita, Water, 9, (2017). doi:10.3390/w9040269

8. ISO, (2009)

9. G. M. Smart, Journal of Hydraulic Engineering, 125 (2), (1999). doi : 10.1061/(ASCE)0733-9429(1999)125:2(106)

10. J. Le Coz, A. Hauet, G. Pierrefeu, G. Dramais, B. Camenen, Journal of Hydrology, 394 (1), 42-52, (2010). doi:10.1016/j.jhydrol.2010.05.049.

11. A. H. Cardoso, W. H. Graf, G. Gust, Journal of Hydraulic Research, 27 (5), (1989). doi: $10.1080 / 00221688909499113$ 